\title{
Geomagnetic 100-ky variation extracted from paleointensity records of the equatorial and North Pacific sediments
}

\author{
Yukiko Yokoyama ${ }^{1}$, Toshitsugu Yamazaki ${ }^{2}$, and Hirokuni Oda ${ }^{2}$ \\ ${ }^{1}$ Department of Biosphere-Geosphere System Science, Faculty of Informatics, Okayama University of Science, Japan \\ ${ }^{2}$ Geological Survey of Japan, AIST, Higashi, Tsukuba, Japan
}

(Received October 27, 2006; Revised May 1, 2007; Accepted May 26, 2007; Online published July 20, 2007)

\begin{abstract}
We have analyzed three sets of paleointensity series varying in duration from 300 to $1600 \mathrm{ka}$ to confirm the existence of paleosecular variation on a 100-ky time scale. The data series are from sediment cores in the equatorial and North Pacific regions. We transformed the three time series into wavelet space and found characteristic variations in a 100-ky time scale. We also calculated wavelet correlation between the series of the paleointensity and rock magnetic parameters of each core to assess the efffect of rock magnetic properties on relative paleointensity. The wavelet correlation coefficients of the three cores are small, so the change in the paleointensity in each core is concluded to be independent of magnetic properties. In order to confirm the cause of the paleointensity variation, we calculated the wavelet correlation among the three cores. The relative intensity variations of the three cores are significantly correlated, while rock magnetic parameters are not, indicating that there are differences in rock magnetic variations among the cores, although they appear to occur on similar scales. On the other hand, all sets of relative paleointensity have synchronous variations. Such paleointensity variations observed over a wide area are considered to originate from changes in the Earth's magnetic field.
\end{abstract}

Key words: Paleosecular variation, paleointensity, geodynamo, Earth's orbit, excentricity, geomagnetic field, secular variation.

\section{Introduction}

The Earth's main geomagnetic field is generated by a fluid motion of the iron liquid core. The source of the motion is believed to be the thermal and gravitational energy released by the cooling Earth (e.g., Jacobs, 1987). However, paleomagnetic studies carried out within the past decade have indicated the possibility of a different energy source: continuous records of paleomagnetic inclination and relative paleointensity from ocean sediment cores show variations with time scales close to those of the Earth's orbit and climate, that is, 40 and $100 \mathrm{ky}$ (Channell et al., 1998; Yamazaki, 1999; Yokoyama and Yamazaki, 2000; Yamazaki and Oda, 2002; Yamazaki and Oda, 2004; Thouveny et al., 2004).

In contrast, other researchers have proposed that the long time scale variations are caused by changes in rock magnetic properties (e.g., Guyodo et al., 2000; Roberts et al., 2003). Their conclusions are mainly derived from two observations. One is that some investigations have failed to find the characteristic variation in the paleomagnetic records; the other is that rock magnetic parameters change on time scales similar to paleomagnetic intensity variations, making it difficult to determine the significance of the characteristic signals even when they are found.

However, these observations could arise from improper time series analysis methodolgies and/or an insufficient

Copyright (c) The Society of Geomagnetism and Earth, Planetary and Space Sciences (SGEPSS); The Seismological Society of Japan; The Volcanological Society of Japan; The Geodetic Society of Japan; The Japanese Society for Planetary Sciences; TERRAPUB number of analyses. Some of researchers who failed to find the characteristic variation in paleomagnetic records used a stationary analysis method, which is believed to be unsuitable for the purpose (e.g., Guyodo and Valet, 1999). Paleomagnetic records should be treated as non-stationary series for the following two reasons. The first is that the longscale variations should be basically non-stationary if they are caused by changes in orbital parameters or climate. The second is that paleomagnetic records usually include uncertainty in age control. Hence, signals become weak and difficult to detect when records are analyzed by a stationary analysis method.

In carrying out their investigations, some of researchers have neglected the necessary information and thus failed to separate paleomagnetic intensity variations from rock magnetic variations even though the signals had fortunately been detected by a stationary analysis (e.g., Channell et al., 1998). In general, time series contain three kinds of important information, strength, time scale, and position in time space, which relate to amplitude, period, and phase of a periodic change, respectively. If one of the three is neglected, a wrong conclusion may be reached. For example, by looking at a white power spectrum we cannot conclude that the change is of a random process. This is because a white spectrum might be that of an impulse signal, which is extremely different from a random signal. Phase information must additionally be considered in order to distinguish them. Also, in a non-stationary case, we should use the information of position in time space as well as that of time scale.

By taking into consideration the points mentioned above, 
(a)

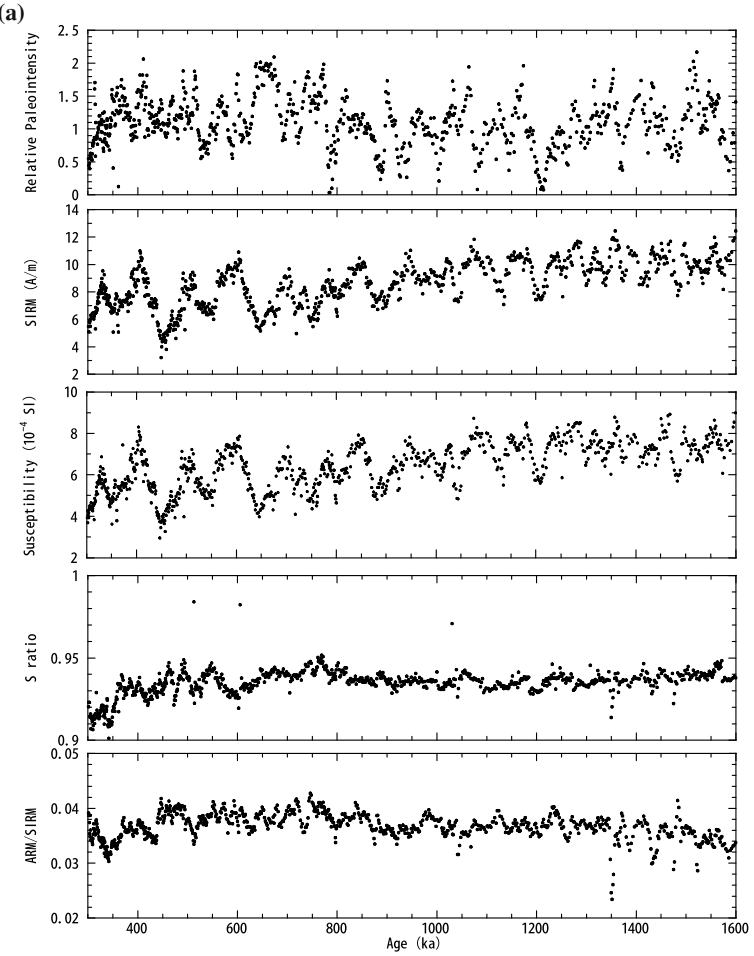

Fig. 1. Time series of relative paleointensity and rock magnetic parameters from the cores (a) MD982185, (b) MD982187, and (c) KR0310-PC1.

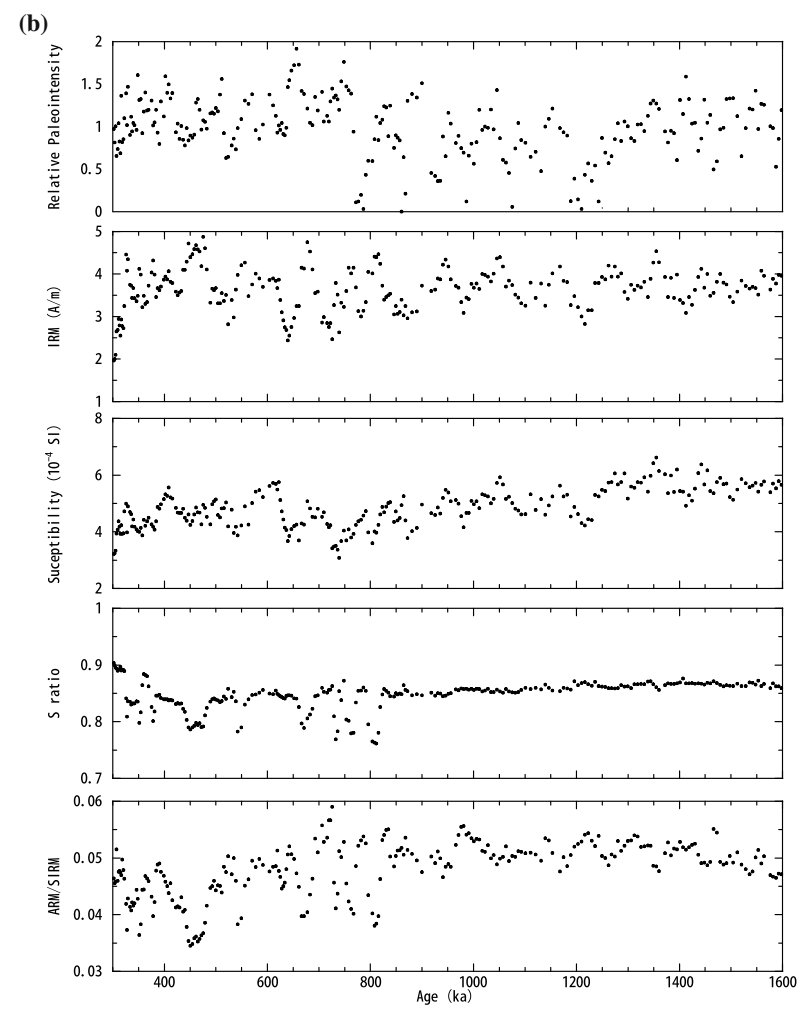

Fig. 1. (continued).

Yokoyama and Yamazaki (2000) (hereafter, Paper I) succeeded in extracting characteristic geomagnetic intensity variation on a 100-ky time scale. Three factors contributed to their success. The first is that a non-stationary time series (c)

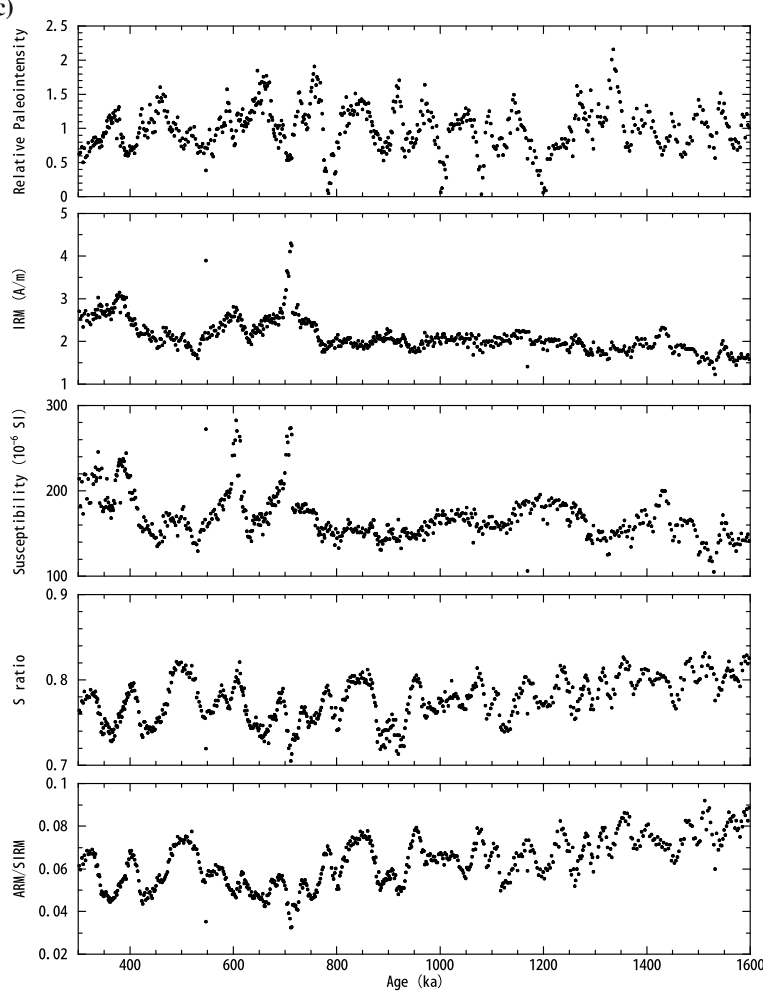

Fig. 1. (continued).

analysis method, the wavelet analysis, was employed (see, for example, Chui (1992) for details of the wavelet analysis). The second is that all three kinds of information of time series were used. The last point is the selection of an appropriate mother wavelet. When an improper mother wavelet is selected, signals become weak and difficult to detect. Hence, in Paper I, we selected the mother wavelet that has characteristics similar to paleomagnetic records from sediment cores (see Section 3 for details), having simple and sharpened waveform and compact support, which roughly corresponds to strong locality in the time domain.

Using the method with the advantages mentioned above, we succeeded in finding the 100-ky variation of the geomagnetic field during the last $700 \mathrm{ky}$ in Paper I. We report here our analysis of the records of a longer duration, from 300 to $1600 \mathrm{ka}$, and confirm that the characteristic variation has continued.

\section{Data}

We analyzed the data sets from the equatorial Pacific sediment cores MD982185 $\left(3^{\circ} 05^{\prime} \mathrm{N}, 135^{\circ} 01^{\prime} \mathrm{E}\right)$ (Yamazaki and Oda, 2002) and MD982187 (4 $\left.4^{\circ} 16^{\prime} \mathrm{N}, 134^{\circ} 49^{\prime} \mathrm{E}\right)$ (Yamazaki and Oda, 2005) and from the North Pacific sediment core KR0310-PC1 $\left(35^{\circ} 15^{\prime} \mathrm{N}, 175^{\circ} 00^{\prime} \mathrm{E}\right)$ (Yamazaki and Kanamatsu, 2007). The data set of each core includes relative paleointensity and four rock magnetic parameters, isothermal remanent magnetization (IRM), magnetic susceptibility, $S$ ratio $\left(S_{-0.1 T}\right)$, and the ratio of anhysteretic remanent magnetization (ARM) to saturation IRM (SIRM).

The physical meanings of the above rock magnetic parameters are as follows. Magnetic susceptibility and IRM are predominantly controlled by the concentration of mag- 


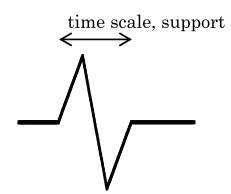

(a)

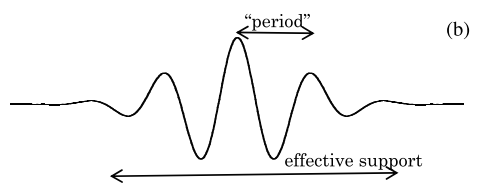

Fig. 2. (a) Mother wavelet used for the wavelet transform in Yokoyama and Yamazaki (2000) and this study. The width of the arrow indicates the time scale of the wavelet, which is equal to the length of wavelet support. (b) Morlet wavelet used for other analyses (Guyodo et al., 2000; Horng et al., 2003; Roberts et al., 2003). The effective width is shorter than the theoretical support, which is infinity, and consists of several times of the scale index called "period".

netic minerals in the sediments, although they are also affected by magnetic grain size (domain state) and a paramagnetic component may contribute to the former. The parameter $\mathrm{S}$ ratio is a proxy of magnetic mineralogy; a lower $S$ ratio indicates that a larger proportion of SIRM is carried by high-coercivity minerals. The definition of Bloemendal et al. (1992) was used for the calculation of $\mathrm{S}$ ratio. As the backfield of $0.1 \mathrm{~T}$ was used here $\left(\mathrm{S}_{-0.1 \mathrm{~T}}\right)$, the "high-coercivity" component not only stands for hematites and goethites but also includes a portion of magnetites, depending on their shape, size, and oxidation state. The ratio of ARM to SIRM (or magnetic susceptibility) is often interpreted as a proxy of magnetic grain size; the average size is relatively larger when ARM/SIRM is smaller (Banerjee et al., 1981; Evans and Heller, 2003). Alternatively, the ratio reflects variations in the strength of magnetostatic interaction among magnetic minerals when the magnetic concentration changes, because ARM is very sensitive to magnetostatic interaction (Sugiura, 1979; Yamazaki and Ioka, 1997).

\section{Analysis Method and Mother Wavelet}

The analyzed records of relative paleointensity and rock magnetic parameters of the three cores are shown in Fig. 1. We interpolated each data series into 2-ky intervals, and obtained the series from 302 to $1608 \mathrm{ka}$. We then transformed the series into wavelet space in a manner similar to that reported in Paper I after removing the linear trend. Following the wavelet transform, wavelet amplitudes were normalized by the maximum absolute value among them.

We used the mother wavelet shown in Fig. 2(a) for the wavelet transform. The reason for the selection of the mother wavelet is as follows. In terms of the signal to be detected in this study, information of the position in time is necessary for the identification of the cause of relative paleointensity variation. In wavelet analysis, a trade-off exists between the resolution in time domain and that in frequency domain (Chui, 1992); therefore, we weighted the time resolution of the wavelet analysis to extract clear information of the position in time.

In order to obtain a higher resolution in the time domain, a compact support mother wavelet is preferred, and the
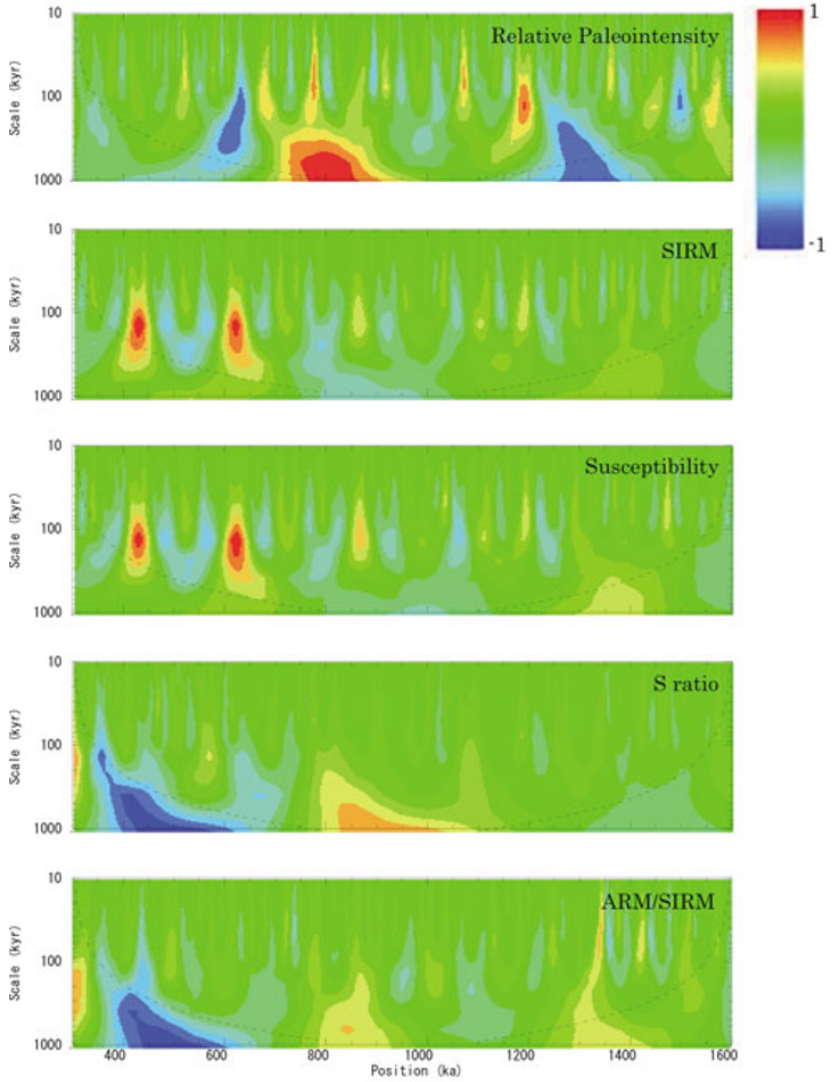

Fig. 3. Wavelet-transformed series of relative paleointensity and rock magnetic parameters from the core MD982185. Broken line indicates the regions where edge effects may appear.

compact support accompanies discontinuities in the waveform of a mother wavelet. Among the compact support mother wavelets, the Haar function is the simplest one. This has zero-order discontinuity, which emphasizes highfrequency noise (see Appendix A for details). The mother wavelet used in this study has first-order discontinuity, and some cardinal B-spline functions have higher-order discontinuity. These functions, which have similar spectrums, are expected to give similar results from the analyses. However, an interpretation of the results of the spline functions is complex due to the complexity of their waveforms. We thus selected the mother wavelet that has a simple waveform (Fig. 2(a)).

\section{Results of Wavelet Transform}

The results of the wavelet transform are shown in Figs. 35. The vertical axis indicates wavelet time scale, which corresponds to the length of wavelet support in this case. The horizontal axis indicates wavelet position in time space. The position at the center of the signal can be considered to represent the age in most cases, but it may sometimes be shifted by about a quarter of the wavelet scale.

The results of the wavelet transform of MD982185 are shown in Fig. 3. In relative paleointensity, a long-scale variation of several hundred kiloyears is the clearest signal. Pairs of strong signals appear around 700 and $1250 \mathrm{ka}$. This signal has a longer time scale than several hundred kiloyears, although only two sets of the signal were detected 

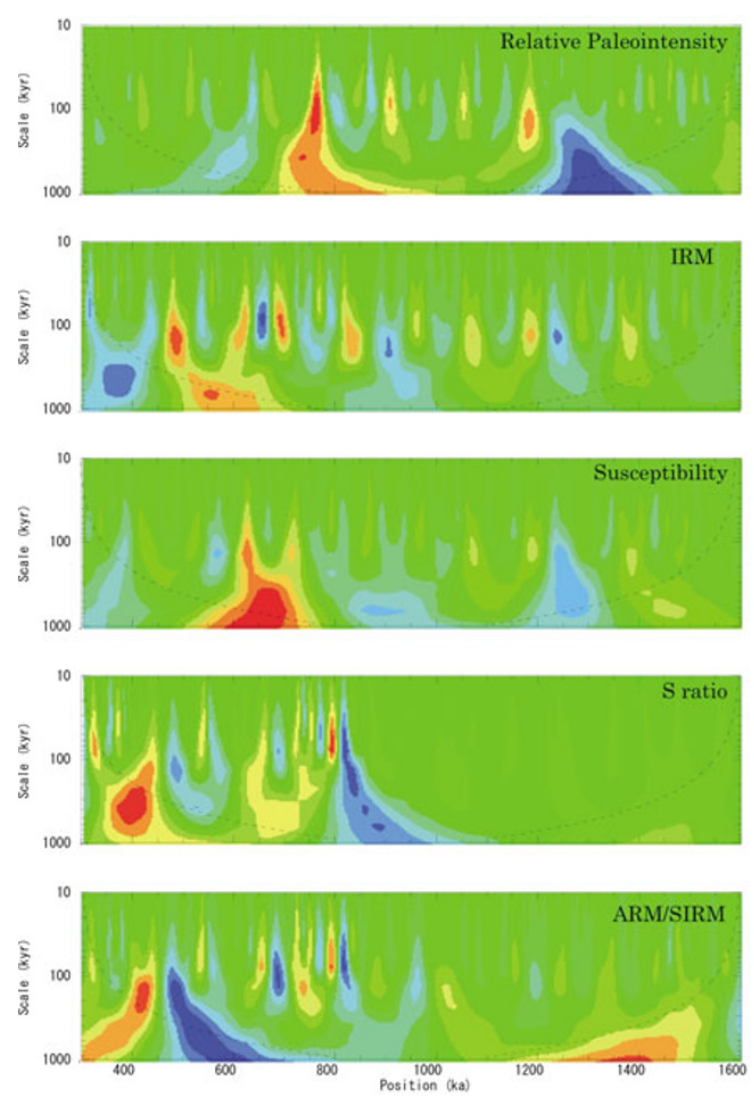

Fig. 4. Wavelet-transformed series of relative paleointensity and rock magnetic parameters from the core MD982187.

because of the limitation in data length. Hence, this time scale will not be argued here.

The second clearest signal is the variation on a scale of $100 \mathrm{ky}$. Positive and negative peaks are repeated around the 100-ky scale. Wavelet signal patterns on this time scale are elongated into a wide time scale, mainly due to the properties of the mother wavelet, which has a low resolution in frequency domain and a high resolution in time domain. Because a wavelet scale is regarded to be different when it differs by a factor greater than two (Chui, 1992), the signal can be spread from 50 to $200 \mathrm{ky}$ in the time-scale domain. In Fig. 3, the actual signal seems to spread into a time scale shorter than $50 \mathrm{ky}$ due to the fact that the signal in the time series contains sharpened waveforms which the mother wavelet is sensitive to. While the time scale of the signal is broad, the signal pattern is localized in the time domain and repeated many times. The temporal interval of the repeated signal pattern is relatively stable at about 100 ky. Hence, we subsequently refer to the variation with such a signal pattern as the 100-ky variation.

Rock magnetic parameters SIRM and susceptibility show quite similar wavelet patterns, as expected from the fact that both are mainly controlled by the magnetic mineral concentration. Similar to relative paleointensity, both display the 100-ky variation, although they have strong signal sets of positive-negative-positive peaks during 400-600 ka. The wavelet patterns of S ratio and ARM/SIRM resemble each other, with both showing strong long-scale signals and an indistinct $100-\mathrm{ky}$ variation.
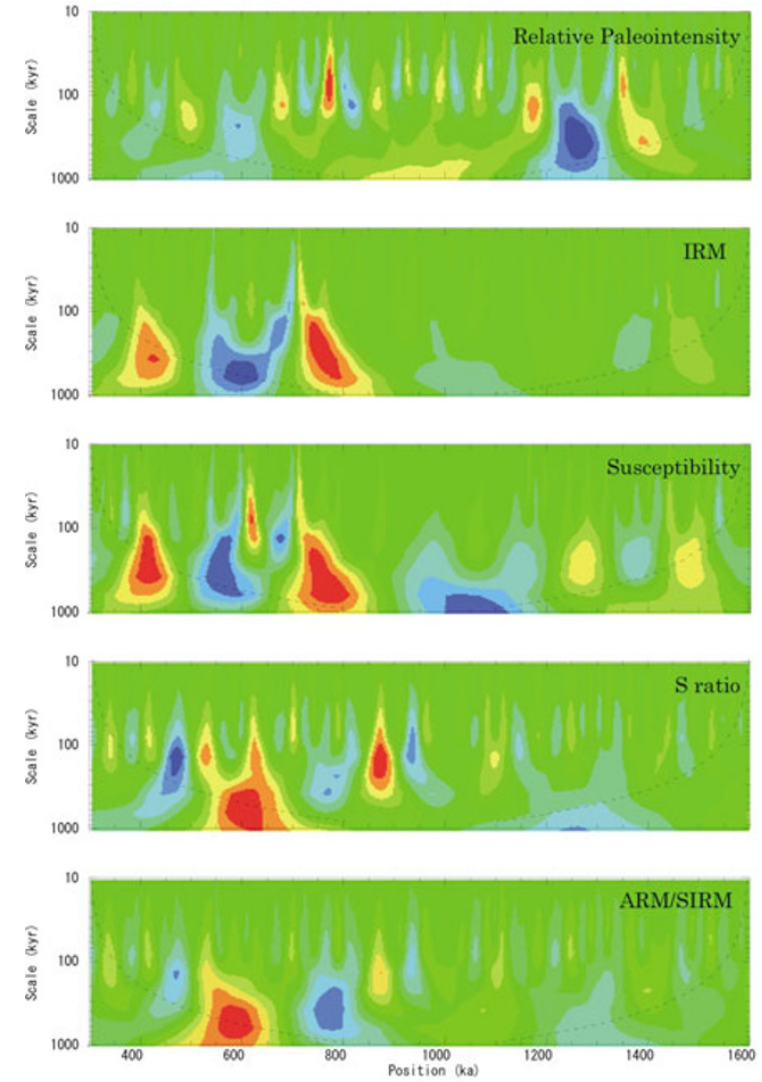

Fig. 5. Wavelet-transformed series of relative paleointensity and rock magnetic parameters from the core KR0310-PC1.

The results of the wavelet transform of MD982187 are shown in Fig. 4. The wavelet pattern of relative paleointensity closely resembles that of MD982185 (Fig. 3), and the longer scale and the 100-ky variations are clearly observed. The rock magnetic parameters IRM and susceptibility show different patterns after $800 \mathrm{ka}$ and a similar pattern before that. Both patterns are different from that of relative paleointensity. The parameters $S$ ratio and ARM/SIRM show similar patterns, which are different from that of relative intensity.

Figure 5 shows the results of KR0310-PC1. Relative paleointensity has a long-scale wavelet pattern that is slightly different from those of MD982185 and MD982187. On the other hand, the 100-ky variation closely resembles those of MD982185 and MD982187. Also, in this case, IRM and susceptibility show similar wavelet patterns. The 100-ky variation is not clear in either of these parameters. The parameters S ratio and ARM/SIRM also show similar patterns. Long-scale variations are clear after $800 \mathrm{ka}$, and the 100 -ky variations are observed throughout the length of the data.

\section{100-ky Variation in Relative Intensity}

In the previous section, the $100-\mathrm{ky}$ variation in relative paleointensity was detected using the wavelet analysis. In this section, we show the variations in frequency domain through a comparison of the variations on neighboring time scales. Figure 6 shows the extracted series of wavelet amplitude on scales of 50, 100, and $200 \mathrm{ky}$. These are cross- 


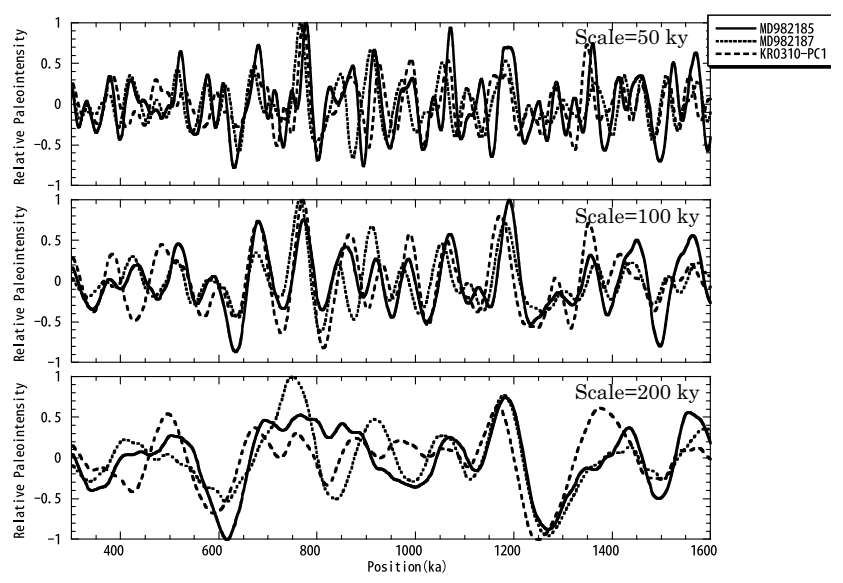

Fig. 6. Wavelet-amplitude variations of relative paleointensity on time scales of 50,100, and $200 \mathrm{ky}$. Amplitude is normalized by the maximum absolute value.

sections of Fig. 3-5 along the time scales, with the amplitudes normalized by the respective maximum absolute values.

In terms of the variation on the time scale of $50 \mathrm{ky}$, most intervals between peaks are several ten kiloyears, as expected. Peak intervals on the scale of $100 \mathrm{ky}$ are roughly twice that in the case of $50 \mathrm{ky}$, and most peaks seem to correspond with the pairs of peaks in the 50-ky time scale. Such patterns are often observed in the presence of a sharpened waveform; peaks of wavelet amplitude series stay in similar position in time space but they are split as the time scale decreases. Hence, such patterns observed in 50- and 100 -ky time scales indicate sharpened waveforms of the extracted signal. In other words, the extracted signals in both scales are mostly identical.

This identification is confirmed in frequency domain. We calculated the power spectra of the wavelet amplitude series in both time scales using the discrete Fourier transform (DFT) with the orthogonal basis set (e.g., Oppenheim and Schafer, 1975). The result is shown on the right-hand side of Fig. 7. All three cores show two strong spectrum peaks around the 50- and 80-ky periods in the case of the 50-ky time scale. The spectrum peaks of the longer period mostly overlap with the shortest spectrum peak on the 100-ky scale.

In the case of the 200-ky time scale in Fig. 6, there are two large-amplitude troughs around 600 and $1250 \mathrm{ka}$, which are not distinct in the 100-ky time scale. This implies that these two troughs reflect a different phenomenon from the variation in the $100-\mathrm{ky}$ time scale. Most of the other peaks in the 200-ky time scale seem to be a change of amplitude of peaks in the 100-ky time scale. In summary, the wavelet amplitude series in the 200-ky time scale includes two different kinds of signals - the 100-ky variation and another, longer scale variation.

The power spectra in the case of the 200-ky time scale (Fig. 7) display three strong peaks around the 120-, 200-, and 300-ky period in all three cores. The shortest spectrum peak corresponds well to the one in the 100-ky time scale; the other two peaks appear at similar periods as those in the 100-ky scale, but they are much weaker. Hence, we consider the two peaks in scale $200 \mathrm{ky}$ to be associated with
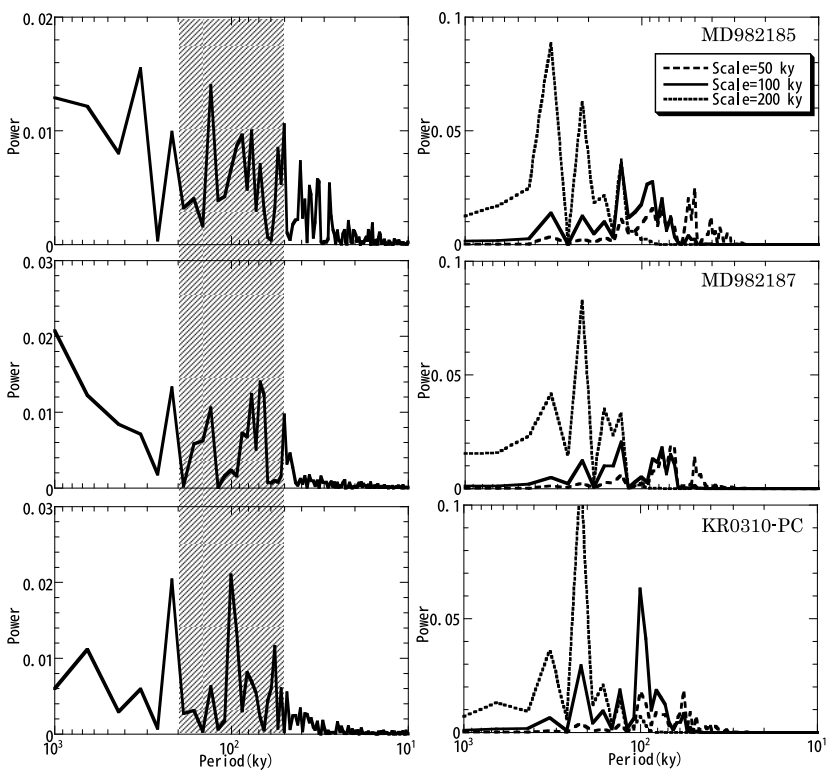

Fig. 7. Power spectra of the relative paleointensity series throughout the entire duration (left), and those of wavelet amplitude variations on time scales of 50, 100, and $200 \mathrm{ky}$ (right).

the two large troughs observed in Fig. 6 and conclude that the $100-\mathrm{ky}$ variation is distributed mainly from the 50 - to 200-ky period.

The values on the left-hand side of Fig. 7 show the power spectrum of the relative intensity series for the entire duration. Because the power spectrum was calculated by DFT with an orthogonal basis set, a power is conserved in this case, while was not the case in our wavelet analysis. A shaded area on Fig. 7 represents a region of the 100-ky variation, and the spectra in this region have similar shapes to those on the right-hand side of Fig. 7. However, their amplitudes are different because our wavelet analysis overestimates the power and because the wavelet amplitudes are normalized.

In order to estimate the power of the 100-ky variation, we have summed the powers of the relative intensity series in the shaded region. The power of the 100-ky variation in ratio is calculated as $0.41,0.47$, and 0.51 in the cases of MD982185, MD982187, and KR0310-PC1, respectively. We also examined the temporal change of the power of the $100-\mathrm{ky}$ variation by calculating the ratio of the power with a 400-ky sliding window, as shown in Fig. 8. Powers of periods shorter than $50 \mathrm{ky}$ and those longer than $200 \mathrm{ky}$ are also calculated for comparison. Through the analyzed duration, a power ratio of 100-ky variation is roughly compatible with that of the shorter period in MD982185. In MD982187 and KR0310-PC1, the power is dominant in most durations. The ratios of the three cores range roughly from 0.2 to 0.8 , which is interpreted as corresponding to the range from 0.45 to 0.89 in the amplitude dimension. The amplitude of the 100 -ky variation thus continuously occupies more than $45 \%$ of the relative intensity for the entire duration, implying that the 100-ky variation continues throughout the analyzed duration. 


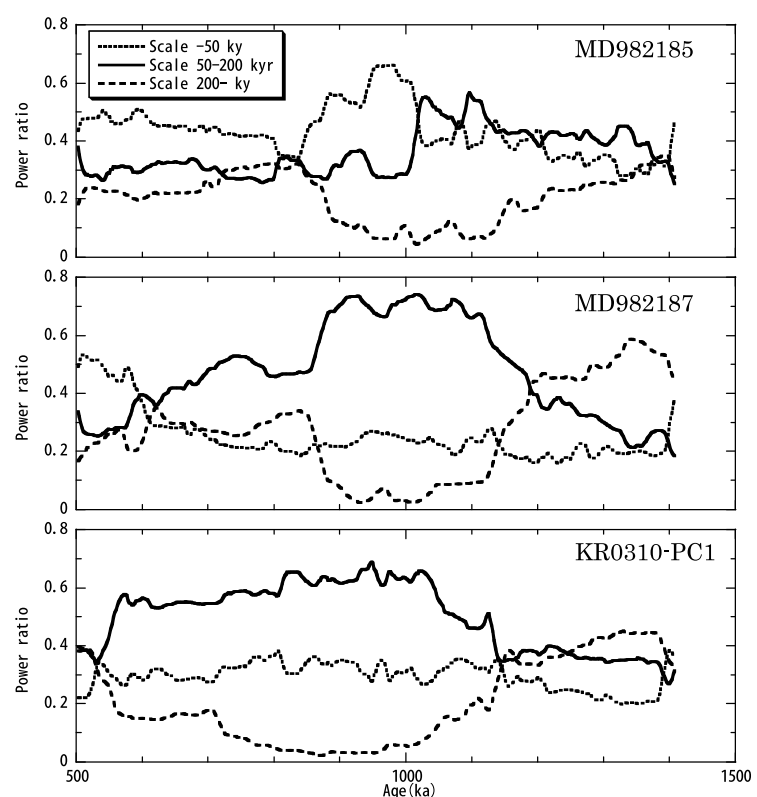

Fig. 8. Temporal change in the power ratio of the 100-ky variation in comparison with those with a time scale shorter than $50 \mathrm{ky}$ and longer than $200 \mathrm{ky}$.
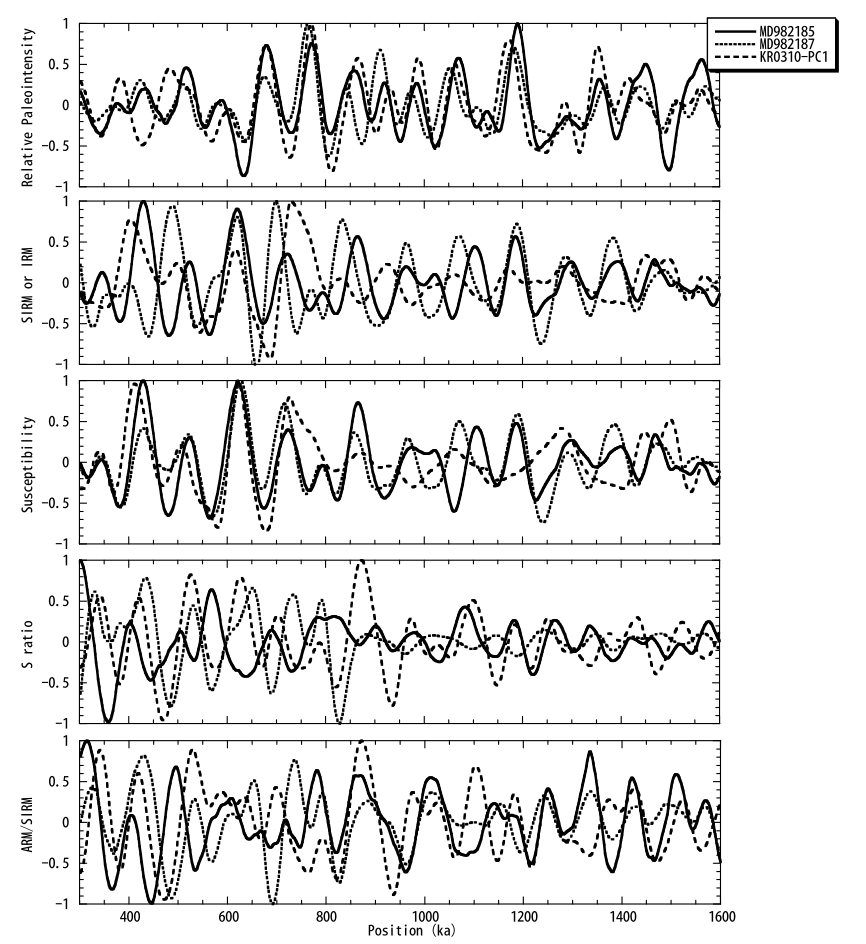

Fig. 9. Wavelet amplitude variations of relative paleointensity and rock magnetic parameters on a time scale of $100 \mathrm{ky}$.

\section{Wavelet Correlations between Relative Pale- ointensity and Rock Magnetic Parameters}

In the previous section, we showed that the wavelet patterns of relative paleointensity and those of rock magnetism are different. In this section, we examine further this difference by focusing on the 100-ky time scale. Our purpose is to demonstrate that the 100-ky variation in relative paleointensity is independent of variations in rock magnetic parameters.

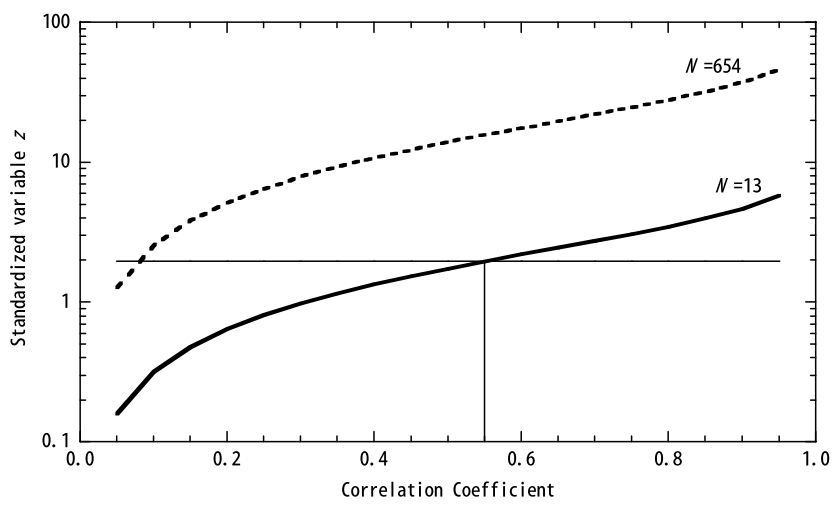

Fig. 10. Relation between standardized variable $z$ of normal distribution and correlation coefficient. When sample number $N=13, z$ becomes 1.96 with correlation coefficient of 0.55 .

We first extracted the 100-ky wavelet components of relative intensity and rock magnetic parameters as a time series, as shown in Fig. 9. We then calculated the correlation coefficients between the time series of relative intensity and rock magnetic parameters. If the two series are synchronous and have similar wave-forms throughout the duration, their correlation coefficient should be large. Conversely, if the two series are not synchronous, their correlation coefficient should be small, regardless of their common time scale. In other words, we can assess whether the two series are directly linked based on the correlation coefficient for entire duration. For comparison purposes, we also calculated the correlation coefficients for the 50- and 200-ky time scales, which are neighboring ones to $100 \mathrm{ky}$.

For the correlation coefficients, we adopted the value of 0.55 as the criteria for determining the presence of a significant correlation. When random error is assumed, the correlation coefficient $r$ is transformed into the standardized variable $z$ of normal distribution, according to

$$
z=\frac{\sqrt{N-3}}{2} \ln \left[\frac{1+r}{1-r}\right]
$$

(Bendat and Piersol, 1986). The sample number $N$ is 654 $(=(1608 \mathrm{ka}-302 \mathrm{ka}) / 2 \mathrm{ky}+1)$ in this study, although it may be calculated as $13(=654 / 50)$, when we consider our target period, $100 \mathrm{ky}$, which consists of 50 samples. In order to reject the hypothesis that the two series are not correlated at the $95 \%$ level, $z$ should be larger than 1.96. This condition is satisfied when $r$ is larger than 0.55 in the case of $N=13$, as shown in Fig. 10.

The calculated correlation coefficients of each core are shown in Table 1. The underlined numbers indicate a significant correlation.

In MD982185, no rock magnetic parameter correlates with relative paleointensity. Between rock magnetic parameters, there is a correlation between SIRM and susceptibility, as expected. This correlation is found in all three scales. The other two rock magnetic parameters, $\mathrm{S}$ ratio and ARM/SIRM, do not show a significant correlation although they do display similar patterns in a wavelet space (Fig. 3). Their similarity is mainly on longer scales and not on those around $100 \mathrm{ky}$. 
Table 1. Wavelet correlation coefficients of each core.

\begin{tabular}{|c|c|c|c|c|c|c|}
\hline Core & Scale (ky) & & Paleointensity & SIRM or IRM & Susceptibility & $S$ ratio \\
\hline \multirow[t]{10}{*}{ MD982185 } & 50 & SIRM & 0.18 & & & \\
\hline & & Susceptibility & 0.15 & $\underline{0.96}$ & & \\
\hline & & ARM/SIRM & 0.05 & -0.15 & 0.00 & 0.47 \\
\hline & 100 & SIRM & -0.07 & & & \\
\hline & & Susceptibility & -0.10 & $\underline{0.98}$ & & \\
\hline & & ARM/SIRM & -0.02 & -0.16 & -0.03 & 0.39 \\
\hline & 200 & SIRM & -0.26 & & & \\
\hline & & Susceptibility & -0.29 & 0.96 & & \\
\hline & & $\mathrm{S}$ ratio & 0.23 & $\overline{-0.40}$ & -0.30 & \\
\hline & & ARM/SIRM & -0.11 & -0.25 & -0.06 & 0.52 \\
\hline \multirow{9}{*}{ MD982187 } & & ARM/SIRM & -0.19 & $\overline{-0.74}$ & 0.05 & $\underline{0.87}$ \\
\hline & 100 & IRM & 0.09 & & & \\
\hline & & Susceptibility & 0.04 & 0.51 & & \\
\hline & & $\mathrm{S}$ ratio & -0.05 & -0.64 & 0.22 & \\
\hline & & ARM/SIRM & -0.07 & $\overline{-0.71}$ & 0.04 & $\underline{0.86}$ \\
\hline & 200 & IRM & -0.10 & & & \\
\hline & & Susceptibility & 0.28 & $\underline{0.57}$ & & \\
\hline & & $\mathrm{S}$ ratio & 0.22 & $\overline{-0.58}$ & 0.17 & \\
\hline & & ARM/SIRM & 0.10 & $\overline{-0.56}$ & 0.01 & $\underline{0.74}$ \\
\hline \multirow[t]{6}{*}{ KR0310-PC1 } & 50 & IRM & -0.04 & & & \\
\hline & & ARM/SIRM & -0.08 & -0.37 & -0.01 & $\underline{0.90}$ \\
\hline & 200 & IRM & -0.16 & & & \\
\hline & & Susceptibility & -0.40 & $\underline{0.88}$ & & \\
\hline & & $\mathrm{S}$ ratio & -0.12 & $\overline{-0.56}$ & -0.29 & \\
\hline & & ARM/SIRM & -0.06 & $\overline{-0.68}$ & -0.53 & $\underline{0.89}$ \\
\hline
\end{tabular}

In MD982187, no rock magnetic parameter correlates with relative paleointensity, as in the other cases. In terms of the rock magnetic parameters, there are correlations among IRM, S ratio, and ARM/SIRM, although that of IRM is negative. Suceptibility becomes involved when the time scale becomes as long as $200 \mathrm{ky}$, but its correlations with the other parameters are not clear.

In KR0310-PC1, there is again no correlation between a rock magnetic parameter and relative intensity. In terms of the rock magnetic parameters, there are correlations between IRM and susceptibility, and between $S$ ratio and ARM/SIRM. Such correlations are also found in wavelet space. At longer scales, a negative correlation appears between IRM and S ratio, and between IRM and ARM/SIRM.

In summary, relative paleointensity and rock magnetic parameters do not correlate at the scales around $100 \mathrm{ky}$; that is, the relative intensity series in the three cores are not contaminated by rock magnetic components.

With respect to the relation among rock magnetic parameters, the correlation between IRM and magnetic susceptibility is a natural result because both mainly represent the magnetic concentration. The negative correlation between
IRM or magnetic susceptibility and ARM/SIRM can be explained by magnetostatic interaction; larger magnetic concentrations can cause a lower acquisition efficiency of ARM due to stronger magnetostatic interaction and, hence, result in smaller ARM/SIRM. This is discussed in detail for core KR0310-PC1 in the accompanying paper (Yamazaki and Kanamatsu, 2007). The same would apply to the other two cores (MD982185 and MD982187); ARM/SIRM ratios reflect variations in the strength of the magnetostatic interaction rather than changes in the magnetic grain size because the sites of the two cores are also in a pelagic environment where changes in the magnetic grain size are expected to be small.

The cause of the correlation between ARM/SIRM and $S$ ratio is not clear. Yamazaki and Kanamatsu (2007) presents a possible scenario for the central North Pacific (KR0310-PC1): an increase in the eolian component during the glacial periods may result in lower $S$ ratios because the eolian component would have abundant highcoercivity minerals, such as hematite, and the synchronous lower ARM/SIRM may be caused by a larger average grain size of the eolian component and/or a larger proportion of 
Table 2. Wavelet correlation coefficients among three cores.

\begin{tabular}{|c|c|c|c|c|c|c|c|}
\hline & \multirow{2}{*}{$\begin{array}{l}\text { Scale } \\
\text { Core }\end{array}$} & \multicolumn{2}{|c|}{$50 \mathrm{ky}$} & \multicolumn{2}{|c|}{$100 \mathrm{ky}$} & \multicolumn{2}{|c|}{$200 \mathrm{ky}$} \\
\hline & & MD982185 & MD982187 & MD982185 & MD982187 & MD982185 & MD982187 \\
\hline \multirow[t]{2}{*}{ Paleointensity } & MD982187 & 0.40 & & $\underline{0.66}$ & & 0.75 & \\
\hline & KR0310-PC1 & 0.44 & 0.34 & $\underline{0.66}$ & 0.51 & $\underline{0.61}$ & $\underline{0.59}$ \\
\hline \multirow[t]{2}{*}{ SIRM or IRM } & MD982187 & 0.14 & & 0.17 & & 0.13 & \\
\hline & KR0310-PC1 & 0.05 & 0.02 & 0.23 & 0.01 & 0.15 & -0.35 \\
\hline \multirow[t]{2}{*}{ Susceptibility } & MD982187 & 0.52 & & $\underline{0.68}$ & & 0.47 & \\
\hline & KR0310-PC1 & 0.30 & 0.31 & 0.49 & 0.37 & 0.23 & -0.07 \\
\hline \multirow[t]{2}{*}{$\mathrm{S}$ ratio } & MD982187 & & -0.30 & -0.41 & & -0.71 & \\
\hline & KR0310-PC1 & -0.06 & 0.22 & -0.05 & 0.32 & 0.09 & -0.09 \\
\hline \multirow[t]{2}{*}{ ARM/SIRM } & MD982187 & 0.16 & & 0.20 & & 0.01 & \\
\hline & KR0310-PC1 & -0.01 & 0.19 & 0.08 & 0.18 & -0.01 & -0.16 \\
\hline
\end{tabular}

titanomagnetites with ilmenite lamellae of volcanic or plutonic origin, which have stronger magnetic interaction. In the other two cores, the variations of ARM/SIRM and $S$ ratio are very small, but they still are significantly correlated. There is a possibility that a similar mechanism may be at work in these cores, although the supply of the terrigenous component would be by a fluvial process from New Guinea instead of an eolian process.

\section{Wavelet Correlations among Cores}

In the previous sections, we showed that relative paleointensity series of the three cores have the 100 -ky variation that is uncorrelated with rock magnetic parameters. In this section, we examine if the variation is a global phenomenon or not. For this purpose, we have calculated wavelet correlations of the relative paleointensity and rock magnetic parameters among the three cores. Because core KR0310PC1 is separated from the other two cores by a distance of more than $5000 \mathrm{~km}$, a correlation among three cores must indicate that the variation is a global one.

The calculated wavelet correlation coefficients of relative paleointensity are shown in Table 2 . There are correlations between MD982185 and MD982187 and between MD982185 and KR0310-PC1 in the 100-ky scale. The coefficient between MD982187 and KR0310-PC1 is 0.51, slightly smaller than 0.55 , but this core combination is interpreted as also being correlated because the other two combinations show significant correlations. In the 200-ky scale, the correlations among the three cores are clear. This strong correlation is mainly due to the two events around 1600 and $1250 \mathrm{ka}$, which are commonly observed in all three cores (Fig. 6), and partly due to the leakage of the power of the 100-ky variation (Fig. 7).

None of rock magnetic parameters seem to have any correlation among the three cores. This indicates that the changes of the rock magnetic parameters are strongly controlled by local factors even though they have a common origin; the response of the magnetic properties of sediments to climate change differs at different locations.

\section{Discussion and Conclusion}

We extracted the 100-ky variation in relative paleointensity of all three cores and, by calculating their wavelet correlation, clarified that the variation is independent of changes in rock magnetic parameters. In addition, by comparing the variations among the three cores, we found that the relative paleointensity variation is a global phenomenon, while rock magnetic variations are local ones. Hence, we conclude that the 100-ky variation of relative paleointensity originates from changes in the Earth's magnetic field.

Such successful results are based on the three points mentioned in Introduction and to inter-core comparisons. Some previous wavelet analysis studies of paleomagnetic records that did not take at least one of the three points into consideration reported the absence of the 100-ky variation (Guyodo et al., 2000; Roberts et al., 2003; Horng et al., 2003). Roberts et al. (2003) performed wavelet analysis on the inclination records of Yamazaki and Oda (2002); using the Morlet mother wavelet (Fig. 2(b)), they did not find the typical wavelet signal, while using a stationary analysis method, Yamazaki and Oda (2002) extracted the 100-ky variation. The stationary signal should be extracted by the wavelet analysis. This discrepancy can be explained by an intermittent signal in the inclination (see case (iii) of Appendix B for details). The power on the 100-ky "period" is calculated to be low, while shorter "periods" display higher power and cyclic patterns. In the wavelet results of Roberts et al. (2003), we found cyclic patterns near the 20-ky "period" whose cycle seems to be roughly $100 \mathrm{ky}$. In other words, the signal found by Yamazaki and Oda (2002) also appears in this wavelet result, but in an unexpected form: the signal is weak and appears in short "periods" due to the improper selection of a mother wavelet.

Horng et al. (2003) also used the Morlet wavelet for the analysis of paleomagnetic intensity and inclination records. In terms of the relative paleointensity, most of the power is occupied by periods longer than $200 \mathrm{ky}$. Because of this, shorter-scale components, including the 100-ky scale, could not be clearly detected. This results from the character of the Morlet wavelet normalization. With respect to inclination, a problem similar to the case of Roberts et al. (2003) may reside.

Guyodo et al. (2000) also used the Morlet wavelet for the analysis of the paleointensity record. Despite the problem in their selection of the mother wavelet, they were fortunately able to detect the 100-ky variation, possibly due to the fact that the records have a feature of continuous sharpened waveform (see case (ii) in Appendix B). They also found variations on a similar scale in rock magnetic parameters and calculated their cross-wavelet. The mean value of 
their cross-wavelet corresponds to the wavelet correlation coefficient in our case. The correlation is not necessarily high throughout the entire duration of the records, although the average value may be higher than our cases. However, it must be noted that this does not negate the 100-ky variation because some sites may simply happen to lack any time lag between variations of paleointensity and rock-magnetic properties. In such cases, an existence of the 100-ky variation can be examined by inter-core correlation, as we have shown in this study.

The reports of the wavelet analysis mentioned above thus contain problems in their methods of data analysis. Hence, the existence of the 100-ky geomagnetic variation cannot be denied based on their results.

The time scale found in the geomagnetic variation is close to that of the eccentricity of the Earth's orbit or that of climate changes, as indicated by previous papers (Yamazaki, 1999; Yokoyama and Yamazaki, 2000; Yamazaki and Oda, 2002; Yamazaki and Oda, 2004). We cannot conclude which of the two is responsible for the geomagnetic variation in this paper. We will be able to distinguish a climate origin by comparing the amplitudes ratio of the 40- and 100-ky geomagnetic variations, as discussed in Yamazaki and Oda (2002), because their amplitude ratio in climate temporary changes (Berger et al., 1998). Examination of a 400-ky variation, which is a time scale closely connected with eccentricity, will also be important in this respect. Through such studies, we shall be able to clarify the origin and mechanism of the geomagnetic variation in the near future.

Acknowledgments. We thank M. Torii and T. Hatakeyama for useful discussion. We also thank anonymous referees for reviewing the manuscript.

\section{Appendix A.}

We here show waveform and spectrum for the three kinds of compact support mother wavelets in Fig. A. The Haar function has zero-order discontinuity and has many sidelobes in the spectrum. In other words, this function has a broad spectrum, which results in an emphasis of highfrequency noise in the wavelet analysis. In contrast, the spectrum of the function used in this study, which has firstorder discontinuity, has only small sidelobes, and it is more localized in the frequency domain than the Haar function. The function of spline-2 has the same order of discontinuity, and its spectrum is also localized; hence, the result of the wavelet analysis is expected to be similar to the second one. Similar results are also expected with higher spline- $m$ functions, which have higher order discontinuity and more localized spectra, in the case of smooth signal, although sensitivity to sharpened signal decreases.

In an actual analysis, simple waveform of a mother wavelet is preferred to facilitate the interpretation of the wavelet signal. The Haar function has a pair of positive and negative peaks, and the pair is also present in wavelet space to express a temporal event. Such an image is shown in Fig. A with gray and white rectangles. This also applies to the function adopted in this study. On the other hand, spline- $m$ functions, which have complex waveforms, need
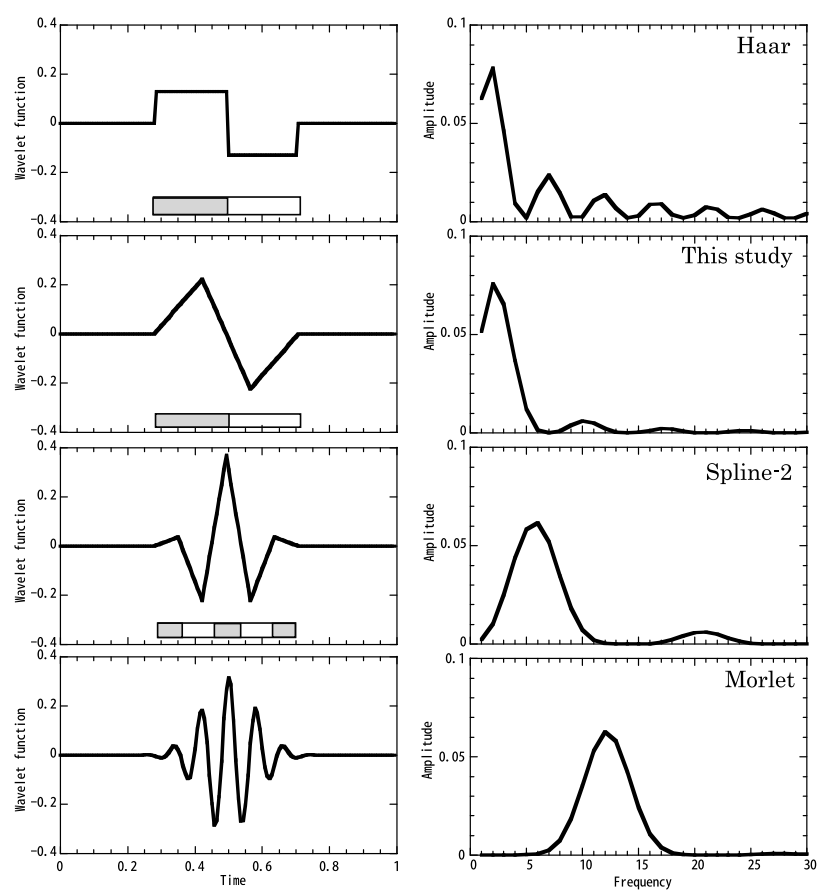

Fig. A. Waveforms (left) and spectra (right) of three kinds of mother wavelets with compact support, Haar function, the function used in this study, and function of spline-2. Total power is normalized. Gray and white rectangles show images of wavelet signal. Morlet wavelet is also shown for comparison.

several or more sets of positive and negative peaks to express an event.

In summary, compact support mother wavelets show a trade-off between locality of spectrum and discontinuity level, which translates into a sensitivity to sharpness and to a simplicity of waveform. These are balanced in the function adopted in this paper.

\section{Appendix B.}

We here show the details of the mother wavelet used in this study. We will first describe the selection criteria of the mother wavelet and then explain its character. We further demonstrate how it works and make a comparison with the Morlet wavelet, which is used in other studies (Guyodo et al., 2000; Roberts et al., 2003; Horng et al., 2003).

Our purpose in this study is to extract useful information from paleomagnetic records obtained from sediment cores. For this, the nature of the records should be considered for a selection of a mother wavelet. The candidates for the physical origin of the change in the records are the Earth's orbit and climate, which are essentially nonstationary phenomena. This makes any associated changes in the records also non-stationary. That the records are recovered from sediment cores is also a distinctive feature; the recovered records include uncertainty in age control and contain high-level noise. The former makes the records apparently non-stationary, while the latter may result in discontinuity because the signal partly disappears into highlevel noise. There is another character obtained from actual records; we found many sharp peaks and troughs (Fig. 1). In summary, the records can be (1) non-stationary, (2) discontinuous, and (3) sharpened. 

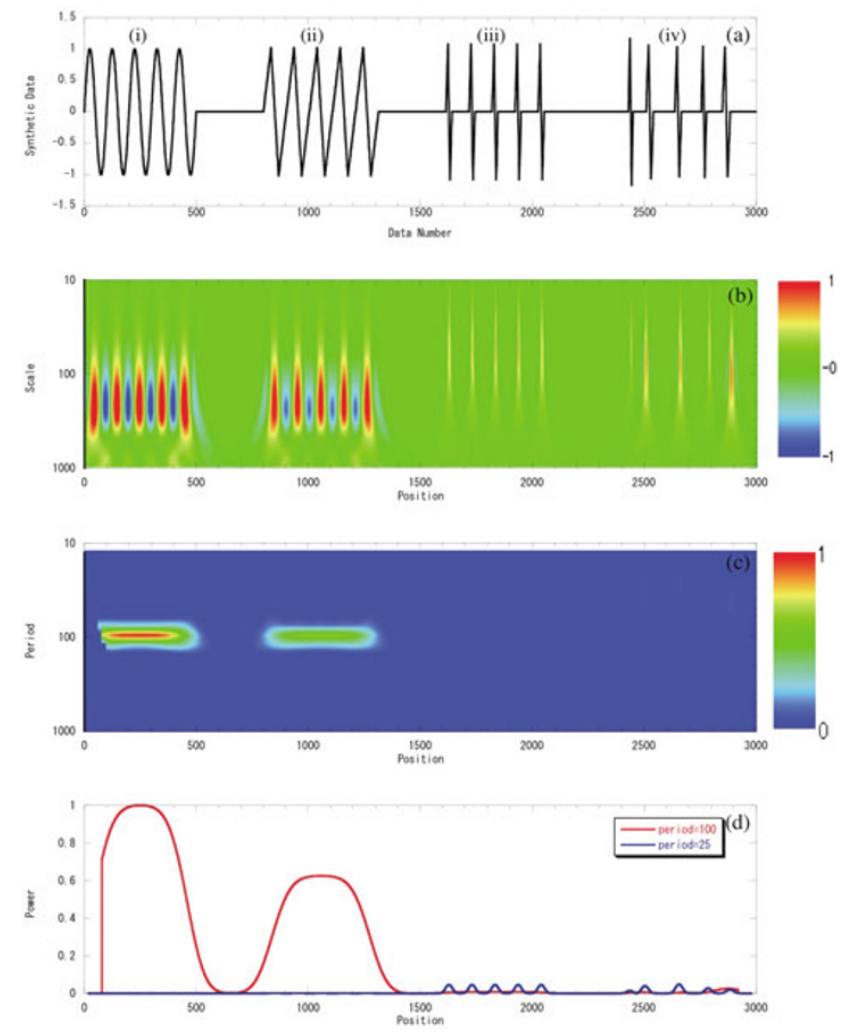

Fig. B. (a) Synthetic time series that is constructed with (i) sinusoids, (ii) chopping wave, (iii) narrow chopping wave with intervals of calm, and (iv) randomly distributed chopping waves. Cycles of the cases from (i) to (iii) is 100 . (b) Wavelet-transformed result using the same mother wavelet as this study. Wavelet amplitude is shown in color contour map. (c) Wavelet-transformed result using the Morlet wavelet. Wavelet power is shown. (d) Wavelet power of the "periods" 100 and 25 extracted from (c).

We selected the mother wavelet in Fig. 2(a) for the analysis of the records with the above features, (1)-(3). The wavelet (a) is compact support and (b) has first-order discontinuity. Owing to character (a), this wavelet works well in cases (1) and (2): power of the wavelet concentrates near the signal, and the signal is detected with strong locality in time domain. Because there is a trade-off between localities in time domain and frequency domain, a strong locality in time domain causes a low resolution in frequency domain. Conversely, when the support is wide, the power of wavelet spreads out widely in time domain and becomes weak, while frequency information is detected with a high resolution. Character (b) gives an advantage in case (3): the discontinuity responds sensitively to sharpened wave form. In other words, broad-frequency spectrum (Fig. A), partly owing to (b), works to an advantage for our data. When a smooth mother wavelet, which has a locality in frequency domain, is used, the detected signal becomes weak.

In order to demonstrate how well the mother wavelet works, we performed a synthetic test. We prepared a synthetic series, which is shown in Fig. B(a). The series consists of four kinds of signals: (i) five cycles of sinusoid, (ii) five cycles of chopping wave, (iii) five cycles of narrow chopping wave with intervals of calm, and (iv) five events of randomly distributed narrow chopping wave with inter- vals of calm. Cycles of the cases from (i) to (iii) is 100, and intervals of the events in (iv) is in the region from 0 to 200. The result of the wavelet analysis is shown in Fig. B(b). Because we weight the information of position in time space in this study, we have plotted the amplitude of wavelet on a contour map. Both information in the time scale and position in time can be seen in this map because our mother wavelet is real. As shown in Fig. B(b), all kinds of signals are clearly detected, even in (i), the smooth and continuous case.

In comparison, we analyzed the same series using the Morlet mother wavelet, whose detailed explanation is shown in Torrence and Compo (1998). This wavelet is basically a complex sinusoidal wave with a Gaussian window and has broad support (Fig. 2(b)) and localized spectrum (Fig. A). The period of the sinusoidal wave is also called "period" in this wavelet analysis. Owing to the features of the spectrum, the function is suitable for analyses of closely cyclic, smooth, and continuous records like the El NiñoSouthern Oscillation (ENSO). We set the wave number of the wavelet as five and the normalization as the square root of wavelet scale, as used in Guyodo et al. (2000). The wavelet power is then calculated in a customary manner, even though information of position in time space is not included in it.

The result is shown in Fig. B(c). The signal of (i) is clearly detected as expected. Power of (ii) is lower than the case of (i) because the smooth mother wavelet is not suitable for the analysis of sharpened wave form. In the cases (iii) and (iv), the power is dramatically reduced and the signals cannot be distinguished. Then, instead of Fig. B(c), we extracted components of "period" 100 and 25, as in Fig. B(d). The power of "period" 100 is as low as to be on the order of 0.1 in case (iii). This low power is due to smoothness, normalization, and wide support of the mother wavelet.

Thus, it has been demonstrated that the Morlet wavelet is not suitable for the analyses of the records with the abovementioned features, (1)-(3). If the normalization is changed by sacrificing physical meaning, energy conservation, signals of the cases (iii) and (iv) will be clearer. In this case, we must note that wavelet signal appears in a shorter "period" than that expected. As shown in Fig. B(d), the power of "period" 25 is stronger than 100 , and there are five peaks. This is because the power of longer "period" tends to leak due to wide support of the mother wavelet. Hence, it is better to adopt the results of the shorter "period". The time scale of the signal can be measured from intervals of the power peaks.

We have therefore been able to sufficiently demonstrate that our mother wavelet is suitable for analyses of paleomagnetic records from sediment cores. We have also demonstrated how our mother wavelet works well, while the Morlet wavelet does not. The importance of the selection of a mother wavelet should not be disregarded.

\section{References}

Banerjee, S. K., J. King, and J. A. Marvin, Rapid method for magnetic granulometry with applications to environmental studies, Geophys. Res. Lett., 8, 333-336, 1981.

Bendat, J. S. and A. G. Piersol, Random Data, Analysis and Measurement Procedures, 566 pp., Wiley-Interscience Pub., New York, 1986. 
Berger, A., M. F. Loutre, and J. L. Melice, Instability of the astronomical periods from 1.5 Myr BP to 0.5 Myr AP, Paleoclimates, 2, 239-280, 1998.

Bloemendal, J., J. W. King, F. R. Hall, and S.-J. Doh, Rock magnetism of Late Neogene and Pleistocene deep-sea sediments: relationship to sediment source, diagenetic processes, and sediment lithology, J. Geophys. Res., 97, 4361-4375, 1992.

Channell, J. E. T., D. A. Hodell, J. McManus, and B. Lehman, Orbital modulation of the Earth's magnetic field intensity, Nature, 394, 464468, 1998.

Chui, C. K., An Introduction to Wavelets, 266 pp., Academic, Boston, 1992.

Evans, M. E. and F. Heller, Environmental Magnetism, 293 pp., Academic Press, 2003.

Guyodo, Y. and J. P. Valet, Global changes in intensity in the Earth's magnetic field during the past $800 \mathrm{ky}$, Nature, 399, 249-252, 1999.

Guyodo, Y., P. Gaillot, and J. E. T. Channell, Wavelet analysis of relative geomagnetic paleointensity at ODP site 983, Earth Planet. Sci. Lett., 184, 109-123, 2000.

Jacobs, J. A ., The Earth's Core, pp. 416, Academic Press, London, 1987.

Horng, C.-S, A. P. Roberts, and W.-T. Liang, A 2.14 Myr astronomically tuned record of relative geomagnetic paleointensity from the western Philippine sea, J. Geophys. Res., 108, doi:10.1029/2001JB001698, 2003.

Oppenheim, A. V. and R. W. Schafer, Digital Signal Processing, 585 pp., Prentice Hall, London, 1975.

Roberts, A. P., M. Winklhofer, W.-T. Liang, and C.-S. Horng, Testing the hypothesis of orbital (eccentricity) influence on Earth's magnetic field, Earth Planet. Sci. Lett., 216, 187-192, 2003.

Sugiura, N., ARM, TRM and magnetic interactions: Concentration dependence, Earth Planet. Sci. Lett., 42, 451-455, 1979.
Thouveny, N., J. Carcaillet, E. Mareno, G. Leduc, and D. Nérini, Geomagnetic moment variation and paleomagnetic excursions since $400 \mathrm{kyr}$ BP: a stacked record from sedimentary sequences of the Portuguese margin, Earth Planet. Sci. Lett., 219, 377-396, 2004.

Torrence, C. and G. Compo, A practical guide to wavelet analysis, Bull. Am. Meteor. Soc., 79, 61-78, 1998.

Yamazaki, T., Relative paleointensity of the geomagnetic field during Bruhnes Chron recorded in North Pacific deep-see sediment cores: Orbital influence?, Earth Planet. Sci. Lett., 169, 23-25, 1999.

Yamazaki, T., and N. Ioka, Cautionary note on magnetic grain-size estimation using the ratio of ARM to magnetic susceptibility, Geophys. Res. Lett., 24, 751-754, 1997.

Yamazaki, T. and T. Kanamatsu, A relative paleointensity record of the geomagnetic field since 1.6 Ma from the North Pacific, Earth Planets Space, 59, this issue, 785-794, 2007.

Yamazaki, T. and H. Oda, Orbital influence on the Earth's magnetic field: 100-ky periodicity in inclination, Science, 295, 2435-2438, 2002.

Yamazaki, T. and H. Oda, Intensity-inclination Correlation for Long-term Secular Variation of the Geomagnetic Field and its Relevance to Persistent Non-dipole Components, AGU Monogr., 145, 287-298, 2004.

Yamazaki, T. and H. Oda, A geomagnetic paleointensity stack between 0.8 and 3.0 Ma from equatorial Pacific sediment cores, Geochem. Geophys. Geosyst., 29, Q11H20, doi:10.2039/2005GC001001, 2005.

Yokoyama, Y. and T. Yamazaki, Geomagnetic paleointensity variation with a 100 ky quasi-period, Earth Planet. Sci. Lett., 181, 7-14, 2000 (Paper I).

Y. Yokoyama (e-mail: yokoyama@big.ous.ac.jp), T. Yamazaki, and H.
Oda 